\title{
White Blood Cell Counting on Smartphone Paper Electrochemical Sensor
}

\author{
Xinhao Wang ${ }^{2,3}+$, Guohong Lin ${ }^{1,2} \ddagger^{*}$, Guangzhe $\mathrm{Cui}^{3}$, Xiangfei Zhou ${ }^{2,3}$, Gang Logan Liu $^{2,3,4_{*}}$ \\ ${ }^{1}$ Department of Immunology, Tongji Medical College, Huazhong University of Science and Technology, Wuhan \\ 430030, China \\ ${ }^{2}$ Micro and Nanotechnology Lab, University of Illinois at Urbana-Champaign, Urbana 61801, USA \\ ${ }^{3}$ Department of Electrical Engineering, University of Illinois at Urbana-Champaign, Urbana 61801, USA \\ ${ }^{4}$ School of Life Science and Technology, Huazhong University of Science and Technology, Wuhan 430074, China \\ $\ddagger$ Equal contributor \\ *Corresponding Authors: Logan Liu loganliu@illinois.edu and Guohong Lin ghlin@illinois.edu
}

\begin{abstract}
White blood cell (WBC) analysis provides rich information in rapid diagnosis of acute bacterial and viral infections as well as chronic disease management. For patients with immune deficiency or leukemia WBC should be persistently monitored. Current WBC counting method relies on bulky instrument and trained personnel and is time consuming. Rapid, low-cost and portable solution is in highly demand for point of care test. Here we demonstrate a label-free smartphone based electrochemical WBC counting device on microporous paper with patterned gold microelectrodes. WBC separated from whole blood was trapped by the paper with microelectrodes. WBC trapped on the paper leads to the ion diffusion blockage on microelectrodes, therefore cell concentration is determined by peak current on the microelectrodes measured by a differential pulse voltammeter and the quantitative results are collected by a smartphone wirelessly within 1 minute. We are able to rapidly quantify WBC concentrations covering the common physiological and pathological range $\left(200 \sim 20000 \mu \mathrm{L}^{-1}\right)$ with only $10 \mu \mathrm{L}$ sample and high repeatability as low as $10 \%$ in $\mathrm{CoV}$ (Coefficient of Variantion). The unique smartphone paper electrochemical sensor ensures fast cell quantification to achieve rapid and low-cost WBC analysis at the point-of-care under resource limited conditions.
\end{abstract}

Keywords: label-free; smartphone; paper electrochemical; white blood cell; diffusion impedance; pointof-care

\section{Introduction}


White blood cell (WBC), also called leukocyte, originates in the bone marrow and circulates throughout the bloodstream. WBC is the main effector cell in the immune system. The main physiology function of WBC is to fight infections from bacteria, viruses, fungal that invade the body. The normal range of $\mathrm{WBC}$ is $4500 \sim 11500 \mu \mathrm{L}^{-1}$ (Rodak et al., 2013) in healthy condition, while in pathology condition, the number will be out of this range. The case when concentration is higher than $11500 \mu \mathrm{L}^{-1}$ is called leukocytosis (Rodak et al., 2013), which is mostly caused by bacteria infection instead of viral infection (Jaffe and Fleisher, 1991; Virkki et al., 2002). Accordingly, WBC is a cell marker to discriminate the early diagnose of bacteria or viral induced infection and impact the therapy decision - the antibiotic therapy or antiviral therapy (Opdenakker et al., 1998). The case when WBC concentration is lower than $4500 \mu \mathrm{L}^{-1}$ is called leukopenia (Rodak et al., 2013), which is commonly caused by medical intervention such as long-term use antibiotics (Granowitz and Brown, 2008) and chemotherapy in cancer (Koinis et al., 2015; Smith et al., 2015). In order to perform safe treatment, WBC concentration needs to be frequently checked to monitor the side effect of chemotherapy and adjust treatment plan, just like glucose management in patient with diabetes mellitus. WBC count provides high diagnosis value in acute infections and chronic disease management. Therefore WBC counting becomes a routine in clinical testing (Rodak et al., 2013) and it is in high demand to have a rapid, easy-to-use, low cost WBC count point of care system in order to meet this significant diagnostic need.

Traditionally WBC counting is done by using hemocytometer under microscope (Hyun et al., 1991) but this method has limited point-of-care applications. Automated high efficiency WBC counting were achieved for clinical use (Buttarello and Plebani, 2008) by measuring the optical scattering (Terstappen et al., 1988), fluorescence labeling (Hudson et al., 1995) or electrical impedance of WBC (Graham, 2003). Such automated hematology analyzer requires bulky instrument and trained operators, which limits the use for point of care applications especially in developing countries with limited resources and thus demands a miniaturized portable hematology analyzer. Several miniaturized platforms for WBC counting have been achieved with microfluidic separation and collection of WBCs however are still lack of system portability because of the use of microscope as detection system (Chen et al., 2008; Cheng et al., 2007; Glynn et al., 2014). WBC counting on microfluidic electrical impedance sensors based on Coulter principle (Graham, 2003) suffers channel-clogging problem and compromised system robustness (Hassan and Bashir, 2014; Holmes et al., 2009). External bulky equipment for liquid pumping and signal acquisition in the aforementioned microfluidic systems limits their application to only laboratory benchtop settings.

Paper (membrane) based liquid sample handling approaches overcome many drawbacks in traditional microfluidic technique and realize pump-free microfluidic flow owing to the capillary force along the paper fibers (Hu et al., 2014; Maxwell et al., 2013). Its low cost, flexibility, ease of patterning 
and disposable properties make this approach a promising candidate for practical point of care applications (Hu et al., 2014; Maxwell et al., 2013). Most paper based microfluidic devices have been coupled with optical colorimetric (Cate et al., 2015) or electrochemical method (Adkins et al., 2015) to quantify ions (Cuartero et al., 2015; Rattanarat et al., 2014) and small molecules such as glucose (Nie et al., 2010), DNA (Li et al., 2015; Liu and Zhang, 2015) or proteins like cancer bio-marker (Ma et al., 2015; $\mathrm{Su}$ et al., 2015). But they suffer from delivery problem limited by pore size when applied to large particles like WBC. Recent attempts were reported to capture WBC on porous membrane substrate by physical trapping (Y. Zhang et al., 2015) or capture antibodies (Shafiee et al., 2015), and quantify using the imaging process with either fluorescent dyes (Shafiee et al., 2015) or gold nanoparticles (Y. Zhang et al., 2015) labelling which adds to the complexity of these attempts. Our work takes the advantages of both the physical property of membrane substrate and ease of electrochemical method at the same time to achieve label-free WBC quantification.

As the most widely used mobile device in the world, smartphone is continuously advanced in computing, information collection and data sharing capabilities, making it an ideal portable receiver by nature for point-of-care applications (Choi, 2016; Damhorst et al., 2015; Zhang and Liu, 2016). Colorimetric sensing using image analysis is one of the most common methods can be applied to paper based applications (Chen et al., 2015; Chun et al., 2014; Delaney et al., 2011; Oncescu et al., 2013; Shen et al., 2012). But owing to variations under different test conditions, more consideration needs to be taken on self-reference and calibration to obtain accurate results (Hong and Chang, 2014). Electrochemical sensing instead is easy to be made compactable to smartphone using audio-jack (Wang et al., 2015), micro-USB (Aggidis et al., 2015) or Bluetooth (Jiang et al., 2014) communication interfaces.

We therefore for the first time propose a paper microfluidic sensor coupled with smartphone electrochemical measurement for label-free WBC counting. The physical trapping of WBC was implemented on our polyvinylidene fluoride (PVDF) membrane with microfabricated interdigitated electrodes and analytical ion probes (ferricyanide/ferrocyanide) were used for electrochemical quantification. EIS (electrochemical impedance spectroscopy) method was often used for such electrode surface analysis on planar solid substrates (Ruan et al., 2002; Varshney and Li, 2009; Yang et al., 2004) but not effectively on porous substrates. Considering the porous property of the membrane electrodes which provides plenty of charge transfer pathways, differential pulse voltammetry (DPV) method was used to quantify diffusion blockage by trapped WBC. Based on our previous efforts on smartphone based platforms for electrochemical sensing (Jiang et al., 2014; Wang et al., 2015; D. Zhang et al., 2015), we implemented a handheld smartphone DPV measurement system through wireless Bluetooth communication and thus made it ready for point-of-care applications. The dynamic range of our portable 
system covers three orders of WBC concentration $\left(10^{2} \sim 10^{4} \mu \mathrm{L}^{-1}\right)$, which enables point-of-care diagnosis even in leukopenia and leukocytosis cases.

\section{Material and Methods}

\subsection{Electrode Fabrication}

Our sensor used three-electrode system for electrochemical testing which was designed using AutoCAD2011. The designed pattern was cut open on planar acrylic material with laser cutting tool (Universal Laser Systems X-600) as shadow mask. The total sensing area for one electrode is about $1 \mathrm{~cm}^{2}$ and the width of interdigitated electrode is $500 \mu \mathrm{m}$. Metal evaporation deposition (CHA SEC-600 E-Beam Evaporator) was used to deposit in a sequence of $10 \mathrm{~nm}$ titanium and $250 \mathrm{~nm}$ gold on PVDF filter

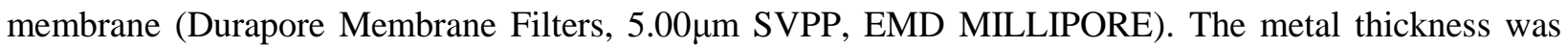
optimized in considering uniform electrode finger resistance and cost effectiveness. Membrane electrode was treated with oxygen plasma (Pico Diener Oxygen Plasma System) for $1 \mathrm{~min}$ before use followed by an activation process. Cyclic voltammetry method was used for this activation process, sweeping from 0.5 to $0.5 \mathrm{~V}$ at the rate of $200 \mathrm{mV} / \mathrm{s}$ for 20 cycles.

\subsection{Cell Preparation}

Cells were cultured and retrieved before electrochemical testing. Hela (ATCC CCL-2) was cultured in DMEM media (Life Technologies, USA) with 10\% fetal bovine serum (LifeTechnologies, Carlsbad, California, USA) in 5\% $\mathrm{CO}_{2}$ at 37 degree. The white blood cell (WBC) was retrieved from healthy human blood (Zen-Bio, Research Triangle Park, North Carolina, USA) by centrifuging. Both Hela cell and WBC were mixed with $1 \mathrm{mM}$ ferricyanide/ferrocyanide in $10 \mathrm{mM}$ PBS solution. The cell number was counted manually using hemocytometer under microscope as reference before testing.

\subsection{Voltammetry Method}

In the electrochemical testing, an advanced potentiostat (CHI660D, $\mathrm{CH}$ Instrument) was used to achieve all the voltammetry methods including CV (cyclic voltammetry), OCV (open circuit voltage), Tafel plot, EIS (electrochemical impedance spectroscopy) and DPV (differential pulse voltammetry). Electrolyte used in these voltammetry methods was chosen to be ferricyanide/ferrocyanide at $1 \mathrm{mM}$ in $10 \mathrm{mM}$ PBS $(\mathrm{pH}=7.35)$ solution. The concentration ratio of ferricyanide and ferrocyanide was controlled at 1 to stabilize potential according to Nernst equation and to be robust to possible evaporation. Electrolyte with too high concentration would make solvent easy to condense out in PVDF membrane especially at low liquid volume. The ratio between analytical ion $\left(\left[\mathrm{Fe}(\mathrm{CN})_{6}\right]^{3-} /\left[\mathrm{Fe}(\mathrm{CN})_{6}\right]^{4-}\right)$ and electrolyte was fixed at 1:10 to make voltammetry method diffusion controlled because of good screening effect of 
electrolyte (Bard et al., 1980). CV was performed at a scan rate of $0.1 \mathrm{~V} / \mathrm{s}$ between $-0.5 \mathrm{~V}$ and $+0.5 \mathrm{~V}$. OCV was performed in a duration time of 20 minutes. Standard $\mathrm{Ag} / \mathrm{AgCl}$ reference electrode (Fisher Scientific) was used when OCV was performed. Tafel plot measurement was executed in a scan rate of $0.1 \mathrm{mV} / \mathrm{s}$ while DPV method was executed with sweeping step at $4 \mathrm{mV}$. The pulse amplitude of DPV was set at $50 \mathrm{mV}$ and the pulse width is $50 \mathrm{~ms}$. EIS was performed at the voltage of $-0.5 \mathrm{~V}$ at the frequency span from $10 \mathrm{~Hz}$ to $100 \mathrm{kHz}$.

\subsection{Simulation}

Finite element method (FEM) was applied to validate sensing principle, which was based on diffusion limited by bio-targets. Electroanalysis module in COMSOL Multiphysics 4.4 was used to explore the change by adding cells in diffusion path (no flux boundary condition). Electroanalytical Butler-Volmer equation was defined as electrode kinetics boundary condition; while the whole domain simulating the region around single finger on interdigitated electrode follow Fick's law of diffusion. Voltage step function and cyclic voltammetry were applied to electrode surface separately to study concentration distribution near electrode surface and current density due to diffusion coefficient variation. The default diffusion coefficient (D) of ferricyanide/ferrocyanide was estimated as $0.75 \times 10^{-5} \mathrm{~cm}^{2} / \mathrm{s}$ (Konopka and McDuffie, 1970); heterogeneous rate constant $\left(k^{0}\right)$ is defined as $4.7 \times 10^{3} \mathrm{~cm} / \mathrm{s}$; transfer coefficient $(\alpha)$ is 0.23 and over-potential is set as $-0.1 \mathrm{~V}$. Parametric sweeping of $\mathrm{D}$ was studied from $0.1 \times 10^{-5} \mathrm{~cm}^{2} / \mathrm{s}$ to $1 \times 10^{-5} \mathrm{~cm}^{2} / \mathrm{s}$ at step size of $0.1 \times 10^{-5} \mathrm{~cm}^{2} / \mathrm{s}$.

\subsection{Portable System}

Portable system design was addressed from electrical and mechanical side. In electrical system, Arduino UNO (ATmega328P) was integrated with external 12-bit DAC (MCP4725) and 16-bit ADC (MCP34265) as analog transmit/receive center. Two operational amplifiers (OPA2378) were built as simplified potentiostat with first-order low pass filter. Sensed signal received by Arduino was finally transmitted through Bluetooth module (HC-05 Bluetooth Serial Module) to smartphone. Maximum value in DPV curve was used for data analysis.

Mechanical design in portable system was implemented by Solidworks 2012. The designed model was printed using 3D printer (Ultimaker 2+, PLA material) at the resolution of $60 \mu \mathrm{m}$. PDMS was mixed with curing agent at the weight ratio of 10:1. After degassing in vacuum desiccator for 30 minutes, PDMS was poured into PLA model and put in oven at 60 centigrade for 12 hours. All the PDMS components were finally assembled together with membrane electrode and wires.

\section{Results and Discussion}




\subsection{Sensing System Design and Principle}

The system diagram of our paper based portable sensing platform was illustrated in Fig. 1a. Voltammetry signal was generated from portable potentiostat after WBC sample was trapped in the membrane (paper) electrode. Electrochemical sensing current during the voltammetry test was retrieved at the same time and transmitted through Bluetooth to smartphone. The smartphone App analyzed the collected data and gave out concentration value of WBC with internal calibration.

The electrochemical sensor head was designed as three gold electrodes which were patterned on a PVDF membrane by e-beam evaporation. (Fig. 1b) Working and counter electrode were designed as interdigitated electrodes to make current flow evenly distributed. Reference electrode was designed closer to working electrode to decrease uncompensated resistance. For the simplicity of fabrication process (Wei et al., 2009), gold was used for all the three electrodes due to its relevant low chemical reactivity and good conductivity. The feasibility of the gold reference electrode was validated by OCV (Open Circuit Voltage) measurement versus standard $\mathrm{Ag} / \mathrm{AgCl}$ reference electrode. The measured coefficient of variation $(\mathrm{CoV})$ of $\mathrm{OCV}$ value was only $0.45 \%$. (Fig. S. 1) PVDF was chosen as porous membrane substrate because of its excellent antioxidation, superior thermal and chemical stability, outstanding physical adsorption as well as good mechanical property (Cao et al., 2006; Sanchuan and Congjie, 1999; Yu et al., 2009). Higher conductivity $\left(4.69 \times 10^{6} \mathrm{~S} / \mathrm{m}\right)$ with thin film metal deposition $(250 \mathrm{~nm})$ was found for PVDF membrane substrate compared with other materials like nitrocellulose membrane (open circuit). The average pore size of PVDF membrane was about $5.0 \mu \mathrm{m}$, smaller than the size of normal human white blood cell $(\sim 10 \mu \mathrm{m})$. The three dimensional porous structure covered with a thin gold layer electrode can provide current amplification. The porous, rough and adsorptive PVDF membrane surface could provide an ideal platform for cell trapping as shown in Fig. 1c.

The electrochemical sensing principle within the membrane electrode was the diffusion blockage effect by the trapped cells which causes a reduced current response (Fig. 1a). This principle could be elucidated by a simple EIS measurement in which an upward bending of diffusion impedance appeared at lower frequencies when more Hela cells were added to the membrane electrode (Fig. 1d). This bending indicated more depletion of ions arriving at electrode (similar to the case with more negative overpotential) primarily owing to cell's capacitive blocking. Such capacitive blocking normally followed by typical Warburg-like part (with slope close to unity) was manifested by the diffusion impedance which was determined by frequency, concentration, diffusion coefficient and over-potential (Bisquert et al., 1999b). To further prove this concept; a simulation was made to show the concentration profile of target oxidant ions around the electrode surface after diffusion for the same duration time (Fig. S. 2a\&b). The cells trapped on or between electrodes blocked ion diffusion pathway and lowered the concentration of target ions reaching the electrode surface. Similar simulation was performed to show the current density 
decrease in $\mathrm{CV}$ due to the lowered concentration resulted from the cell blocked ion diffusion (Fig. S. 2c\&d). It was also noticeable that the change of charge transfer resistance was extremely small, mainly due to the numerous current path ways within porous membrane electrode (Fig. 1d). According to Fick's second law, cell's blockage effect could also be considered as a decrease in effective diffusion coefficient (D). It was illustrated in Juan Bisquert's work that the diffusion coefficient D was a function of diffusion response and diffusion impedance had a negative correlation with the diffusion coefficient (Bisquert et al., 1999a, 1999b, 1998).

The benefit of the porous membrane substrate was studied assuming that the cell trapping could be considered as a change in D. To study both the electrode kinetics and diffusion property of the membrane electrode, Tafel plot measurement was performed as shown in Fig. 1e. The ideal case (no mass transfer effect) for current-overpotential relation can be written as:

$$
i=i_{0}\left[e^{-\alpha f \eta}-e^{(1-\alpha) f \eta}\right] \quad \text { (Bard et al., 1980) }
$$

where $i_{0}$ is exchange current; $\eta$ is over-potential; $f=F / R T$ ( $R$ is gas constant; $T$ is absolute temperature). The slope of cathodic branch in this ideal case is $-\alpha F / 2.3 R T$ even for large over-potential. In reality, this slope will be flattened owing to diffusion limit at very large over-potential indicated by the Tafel plot of a wire electrode in Fig.1e. In comparison, the slope in the Tafel plot of membrane electrode was more flattened even at small over-potential. This phenomenon indicated a relatively strong diffusion limit in membrane electrodes, corresponding to a smaller background diffusion coefficient. The effect of background diffusion coefficient on the sensitivity of cell particle sensing was simulated. (Fig. 1f) Larger diffusion coefficient was observed to lead to higher current density but the current density slope at lower diffusion coefficient was much larger, indicating a more sensitive change with the same amount of variation in diffusion coefficient. Therefore, the membrane electrode with diffusion limit property was proved to be a potential more sensitive platform for particle sensing using diffusion block mechanism.

(Fig. 1 position)

\subsection{Membrane Electrode Characterization}

Double layer capacitance was investigated since it could be key to voltammetry method selection (Bard et al., 1980). The double layer capacitance in our system was estimated according to sensed current and voltage scan rate by cyclic voltammetry method. A comparison of double layer capacitance between wire and membrane electrode was made for electrolyte (10mM PBS) only samples. The double layer capacitance of membrane electrode $(73.5 \mu \mathrm{F})$ was 14.7 times larger than that of wire electrode $(5 \mu \mathrm{F})$. (Fig. 2a) Such large double layer capacitance introduced high interfering charging/discharging current to actual Faradaic current when voltammetry method with high scan rate was applied. This drawback was confirmed with CV method when quantifying Hela cells as shown in Fig. 2b. The sensitivity of CV 
method was quite small and the linear correlation was relatively poor, therefor not appropriate for membrane electrode. On the other hand, differential pulse voltammetry (DPV) is the proper method to effectively reduce non-Faradaic current (double layer capacitor charging/discharging) owning to its current-difference mechanism and very slow scan rate (Bard et al., 1980; Compton and Banks, 2007). Current difference is taken before and after applying a voltage pulse in order to analyze the Faradaic current caused by the small amplitude of pulse. Given the short pulse width $(50 \mathrm{~ms})$ as the constant diffusion response time, the amount of cell particles can be quantified due to different diffusion response on membrane electrode.

The current amplification by the membrane electrode was characterized with respect to a bulk gold wire electrode using cyclic voltammetry method (Fig. 2c). The membrane electrode showed 12.5 times larger peak current than wire electrode. Although the planar surface area of membrane electrode $\left(\sim 25 \mathrm{~mm}^{2}\right)$ was larger than gold wire electrode $\left(\sim 5 \mathrm{~mm}^{2}\right)$, it took only $10 \mu \mathrm{L}$ of sample to generate such high current compared to the minimum liquid needed $(100 \mu \mathrm{L})$ for gold wire electrode. The equivalent current density amplification ratio for membrane electrode was then about 25. The Tafel plot in Fig. 1e showed the membrane electrode had a two orders of magnitude higher exchange current than wire electrode, which can be explained by the large electrode surface area in the following expression:

$$
i_{0}=F A k^{0} C_{O}^{*(1-\alpha)} C_{R}^{* \alpha} \quad \text { (Bard et al., 1980) }
$$

where $\alpha$ is transfer coefficient; $A$ is electrode surface area; $F$ is the Faraday constant; $k^{0}$ is standard heterogeneous rate constant; $C_{O}^{*}$ is bulk oxidant concentration and $C_{R}^{*}$ is bulk reductant concentration.

To ensure membrane electrode can capture cells effectively as illustrated in Fig. 2d, trapping test was made by dropping $\sim 60 \mathrm{k}$ Hela cells in $10 \mu \mathrm{L}$ solution on the membrane and comparing DPV currents before and after washing the electrode (Fig. S. 3). Only 6\% increase in peak current after washing was observed, indicating excellent surface trapping property and advantage in rapid cell captures.

(Fig. 2 position)

Two techniques were used to overcome the hydrophobic property of PVDF and further improve cell trapping. First, PDMS as hydrophobic substrate was attached underneath the membrane electrode to ensure the liquid diffuse only within the membrane first (Fig. S. 4a). Second, oxygen plasma (OP) treatment was also applied to change membrane into hydrophilic, which helped raise the peak current by $35 \%$ (Fig. S. 4b) (Li et al., 2008). Effect of mechanical flexibility of the membrane electrode was also studied and showed excellent stability $(\mathrm{CoV}=1.6 \%)$ in different deformation (Fig. S. 5).

\subsection{Cell Counting on Membrane Electrode}

Hela cell, a human cervical cancer cell, was first used as the surrogate in counting experiments because of its similar size to human WBC. The DPV curve was measured for Hela cells in the amount of 
6 to $600 \mathrm{k}$ in total number in a constant volume of $10 \mu \mathrm{L}$ (Fig. 3a). The reduction current peak was observed near the formal reduction potential of ferricyanide. The minor potential shift could be due to the relative slow sweeping rate $(20 \mathrm{mV} / \mathrm{s})$. The peak current is inversely proportional to the increasing amount of cells, indicating more diffuse pathways were blocked by the cells. A highly linear calibration curve was made between peak current and cell number with a coefficient of determination $\mathrm{R}^{2}=0.994$ (Fig. 3b), a large linear dynamic range covering about 6 orders $\left(10^{1} \sim 10^{6}\right)$ of magnitude of cell numbers, and a limit of detection (LoD) at 60 cells. The DPV curves were analyzed using peak maximum instead of Gaussian peak height because it's more convenient to be realized in mobile application. The feasibility was validatd using Gaussian peak height calibration (Fig. S. 6) as comparison, which showed little effect on linearity and sensing range. The PVDF membrane pore size was investigated and optimized to achieve good linearity (Fig. S. 7). For the case that pore size is larger than target size, the target's diffusion blockage effect will be much less which causes little interference.

After sensor verification using Hela cells, we isolated and counted WBC from the peripheral blood of health individuals. WBC was tested on membrane electrode in the number ranging between $10^{1}$ and $10^{6}$ cells in $10 \mu \mathrm{L}$ liquid samples. Due to the diffusion blockage effect of $\mathrm{WBC}$, the DPV measurements showed a similar inverse proportion between the cell numbers and peak currents (Fig. 3c). The current measurement for cell numbers ranging from $1.5 \mathrm{k}$ to $150 \mathrm{k}\left(150 \sim 15 \mathrm{k} \mu \mathrm{L}^{-1}\right)$ showed a good linearity $\left(\mathrm{R}^{2}=0.938\right)$, exactly covering the WBC concentrations in a healthy person $\left(4500 \sim 11500 \mu \mathrm{L}^{-1}\right)$ (Rodak et al., 2013), leukopenia (decrease in number) and leukocytosis (increase in number) patients (Fox, 1996; Porth, 2011). The WBC testing results suggested the potential for the sensor in clinical diagnosis. It is

worthy to note that some interference may occur in real blood test like red blood cell (RBC) and other small molecules in serum. Since current sensing is based on physical adsorption and diffusion blockage mechanism, small molecules like glucose will cause very little interference (Fig. S. 8) while RBC with similar size to WBC will make a significant difference. Microfluidic cell lysing unit could be integrated as pre-separation stage for real blood portable diagnosis system (Hassan and Bashir, 2014).

(Fig. 3 position)

\subsection{Portable White Blood Cell Counting}

Portable electronic potentiostat system for DPV sensing and Bluetooth communication was described in Fig. 4. The working electrode was stabilized by connecting together with a band-gap reference potential IC (2.5V) to an Op-Amp. Voltage on the reference electrode will be keeping sweeping voltammetry. Pulse width $(50 \mathrm{~ms})$ and sampling time was accurately controlled using the timer function from micro-controller. A 12-bit DAC and 16-bit ADC component were used (Fig. 4a) in order to achieve high current detection resolution as high as $200 \mathrm{nA}$ demonstrated in Fig. S. 9. A $10 \mathrm{k} \Omega$ resistor with $1 \%$ 
tolerance was used to in the current to voltage conversion module in the circuit. Collected voltage values from this simplified potentiostat were transmitted through a wireless Bluetooth module to smartphone. The original DPV curve is plotted on the phone and the measured cell number is calculated according to built-in calibration equation. (Fig. 4b) The membrane electrode was assembled using PDMS as the hydrophobic supporting substrate and was electrically connected with three gold wires to the portable potentiostat. The configuration of the system assembly is shown in Fig. 4c and 4d.

(Fig. 4 position)

The above portable electrochemical sensor was then applied to human WBC counting in the concentration range of $195 \sim 25 \mathrm{k} \mathrm{uL}^{-1}$. The DPV peak current change in this concentration range was linearly proportional to WBC concentration (Fig. 5a). The LoD for our sensing system was $195 \mathrm{uL}^{-1}$, which was 30 times lower than normal concentration $\left(\sim 6000 \mathrm{uL}^{-1}\right)$ in blood. The maximal peak current change $(\sim 60 \mu \mathrm{A})$ was 300 times of the current detection resolution (200nA), which provided a very broad dynamic range for cell concentration measurement. The minimal detectable change in cell concentration can be as low as $2.6 \%$. Normalization by taking the ratio of sample current to background current was performed based on the background current (peak current from electrolyte) to cancel out the variation among different individual membrane electrodes. The normalization method led to a better fitting in calibration. The coefficient of determination $\left(\mathrm{R}^{2}\right)$ for regression fitting was found to be 0.994 throughout the entire concentration range (Fig. 5b). The calibration equation could be expressed as:

$$
\text { Concentration }=10^{116.09-\text { current } / 21.44}
$$

The detection range could be divided into two regions (low and high) which are calibrated separately to get more accurate fitting (Fig. 5c \& Fig. 5d). The CoV in the low concentration was $3.3 \%$ in average while the $\mathrm{CoV}$ in high concentration was $4.3 \%$. The comparison of the measurement result from the portable detection system with commercial potentiostat showed good matching (Fig. S. 10).

(Fig. 5 position)

\subsection{Blood Sample Accuracy Test}

To evaluate the accuracy of portable electrochemical system for WBC counting, 12 processed blood samples of $10 \mu \mathrm{L}$ each from healthy personal with unknown WBC concentration were quantified by both gold standard manual counting method via microscopy and our portable sensing method respectively. (Fig. 6a) The WBC concentration was from $200 \mu \mathrm{L}^{-1}$ to $15000 \mu \mathrm{L}^{-1}$, covering majority clinical relevant disease from leukopenia and leukocytosis. The calibration curve used for quantification was derived from Fig. 5. The overall $\mathrm{CoV}$ of repeatability testing was $8.8 \%$. The $\mathrm{CoV}$ for WBC counting accuracy at low concentration $\left(<2000 \mu \mathrm{L}^{-1}\right)$ was as low as $10 \%$ while that in the high concentration $\left(>8000 \mu \mathrm{L}^{-1}\right)$ was $15 \%$ as shown in Fig. 6. Most data points were distributed around the $y=x$ line with a coefficient of 
determination $\left(\mathrm{R}^{2}\right)$ at 0.993 . The high accuracy and precision of our sensor especially in the low WBC concentration are crucial for applications such as monitoring therapy effects for cancer or immunedeficiency patients (Koinis et al., 2015; Smith et al., 2015). Although slightly lower in very high WBC concentration, the counting accuracy of our sensor is more than sufficient for quick diagnosis of bacterial infection (Jaffe and Fleisher, 1991; Virkki et al., 2002) in which case the WBC concentration increases over 2 folds. In addition, the <1-minute detection time and broad range WBC counting of our portable paper sensor are critical for rapid diagnostics especially in emergency care.

(Fig. 6 position)

\section{Conclusions}

Due to the significance of WBC counting in clinical diagnosis and the highly demand in rapid, low-cost solution for point-of-care WBC test, we demonstrated a novel platform using smartphone based electrochemical method to achieve fast label-free WBC counting on microfabricated membrane electrode. The sensing principle was based on the diffusion blockage by the cells trapped on membrane electrodes and the consequent electrochemical signal change as the function of the cell concentration. The membrane microelectrode showed advantages in current amplification, excellent surface trapping, large double layer capacitance, hydrophilicity and flexibility. By creating a mobile differential pulse voltammeter, the peak currents from tests on WBC at different concentrations were retrieved and transmitted to smartphone through Bluetooth protocol. The across-four-orders-of-magnitude dynamic range for our WBC sensor was able to cover healthy, Leukopenia and Leukocytosis conditions with excellent accuracy and precision. The current system has no cell separation unit which will be integrated in the future for direct whole blood testing. Overall, our mobile WBC counting work showed its potential in future point-of-care applications like inflammation disease diagnosis at home.

\section{Acknowledgements}

This research is partially supported by U.S. Department of Agriculture grant. The authors thank Lisa Plucinski and Wenyue Li for the contribution to Hela cell culturing and Abid Ameen for the help with metal deposition.

\section{References}

Adkins, J., Boehle, K., Henry, C., 2015. Electrophoresis. 1811-1824. 
Aggidis, A.G.A., Newman, J.D., Aggidis, G.A., 2015. Biosens. Bioelectron. 74, 243-262.

Bard, A.J., Faulkner, L.R., Leddy, J., Zoski, C.G., 1980. Electrochemical methods: fundamentals and applications. Wiley New York.

Bisquert, J., Garcia-Belmonte, G., Bueno, P., Longo, E., Bulhoes, L.O.S., 1998. J. Electroanal. Chem. 452, 229-234.

Bisquert, J., Garcia-Belmonte, G., Fabregat-Santiago, F., Bueno, P.R., 1999a. J. Electroanal. Chem. 475, $152-163$.

Bisquert, J., Garcia-Belmonte, G., Fabregat-Santiago, F., Compte, A., 1999b. Electrochem. commun. 1, $429-435$.

Buttarello, M., Plebani, M., 2008. Am. J. Clin. Pathol. 130, 104-116.

Cao, X., Ma, J., Shi, X., Ren, Z., 2006. Appl. Surf. Sci. 253, 2003-2010.

Cate, D.M., Adkins, J.A., Mettakoonpitak, J., Henry, C.S., 2015. Anal. Chem. 87, 19-41.

Chen, W., Long, K.D., Kurniawan, J., Hung, M., Yu, H., Harley, B.A., Cunningham, B.T., 2015. Adv. Opt. Mater. 3, 1623-1632.

Chen, X., Liu, C.C., Li, H., 2008. Sensors Actuators B Chem. 130, 216-221.

Cheng, X., Irimia, D., Dixon, M., Sekine, K., Demirci, U., Zamir, L., Tompkins, R.G., Rodriguez, W., Toner, M., 2007. Lab Chip 7, 170-178.

Choi, S., 2016. Biotechnol. Adv. 34, 321-330.

Chun, H.J., Park, Y.M., Han, Y.D., Jang, Y.H., Yoon, H.C., 2014. BioChip J. 8, 218-226.

Compton, R.G., Banks, C.E., 2007. Understanding voltammetry. World Scientific.

Cuartero, M., Crespo, A., Bakker, E., 2015. Anal. Chem. 87, 1981-1990.

Damhorst, G.L., Duarte-Guevara, C., Chen, W., Ghonge, T., Cunningham, B.T., Bashir, R., 2015. Engineering 1, 324-335.

Delaney, J.L., Hogan, C.F., Tian, J., Shen, W., 2011. Anal. Chem. 83, 1300-1306.

Fox, S.I., 1996. Human Physiology 9th Editon.

Glynn, M.T., Kinahan, D.J., Ducrée, J., 2014. Lab Chip 14, 2844-2851.

Graham, M.D., 2003. J. Assoc. Lab. Autom. 8, 72-81.

Granowitz, E. V, Brown, R.B., 2008. Crit. Care Clin. 24, 421-442.

Hassan, U., Bashir, R., 2014. Lab Chip 14, 4370-4381.

Holmes, D., Pettigrew, D., Reccius, C.H., Gwyer, J.D., van Berkel, C., Holloway, J., Davies, D.E., Morgan, H., 2009. Lab Chip 9, 2881-2889.

Hong, J. Il, Chang, B.-Y., 2014. Lab Chip 14, 1725-1732. 
Hu, J., Wang, S., Wang, L., Li, F., Pingguan-murphy, B., Jian, T., Xu, F., 2014. Biosens. Bioelectron. 54, 585-597.

Hudson, J.C., Brunhouse, R.F., Garrison, C., Rodriguez, C.M., Zwerner, R., Russell, T.R., 1995. Cytometry 22, 150-153.

Hyun, B.H., Gulati, G.L., Ashton, J.K., 1991. Yonsei Med J 32, 283-291.

Jaffe, D.M., Fleisher, G.R., 1991. Pediatrics 87, 670-674.

Jiang, J., Wang, X., Chao, R., Ren, Y., Hu, C., Xu, Z., Liu, G.L., 2014. Sensors Actuators B Chem. 193, 653-659.

Koinis, F., Nintos, G., Georgoulias, V., Kotsakis, A., 2015. Expert Opin. Pharmacother. 16, 1505-1519.

Konopka, S.J., McDuffie, B., 1970. Anal. Chem. 42, 1741-1746.

Li, X., Scida, K., Crooks, R.M., 2015. Anal. Chem. 87, 9009-9015.

Li, X., Tian, J., Nguyen, T., Shen, W., 2008. Anal. Chem. 80, 9131-9134.

Liu, F., Zhang, C., 2015. Sensors Actuators B. Chem. 209, 399-406.

Ma, C., Li, W., Kong, Q., Yang, H., Bian, Z., Song, X., Yu, J., Yan, M., 2015. Biosens. Bioelectron. 63, $7-13$.

Maxwell, E.J., Mazzeo, A.D., Whitesides, G.M., 2013. MRS Bull. 38, 309-314.

Nie, Z., Nijhuis, C.A., Gong, J., Chen, X., Kumachev, A., Martinez, A.W., Narovlyansky, M., Whitesides, G.M., 2010. Lab Chip 10, 477-483.

Oncescu, V., O’Dell, D., Erickson, D., 2013. Lab Chip 13, 3232-3238.

Opdenakker, G., Fibbe, W.E., Van Damme, J., 1998. Immunol. Today 19, 182-189.

Porth, C., 2011. Essentials of pathophysiology: Concepts of altered health states. Lippincott Williams \& Wilkins.

Rattanarat, P., Dungchai, W., Cate, D., Volckens, J., Chailapakul, O., Henry, C.S., 2014. Anal. Chem. 86, $3555-3562$.

Rodak, B.F., Fritsma, G.A., Keohane, E., 2013. Hematology: clinical principles and applications. Elsevier Health Sciences.

Ruan, C., Yang, L., Li, Y., 2002. Anal. Chem. 74, 4814-4820.

Sanchuan, Y., Congjie, G., 1999. Technol. Water Treat. 2.

Shafiee, H., Asghar, W., Inci, F., Yuksekkaya, M., Jahangir, M., Zhang, M.H., Durmus, N.G., Gurkan, U.A., Kuritzkes, D.R., Demirci, U., 2015. Sci. Rep. 5.

Shen, L., Hagen, J.A., Papautsky, I., 2012. Lab Chip 12, 4240-4243.

Smith, T.J., Bohlke, K., Lyman, G.H., Carson, K.R., Crawford, J., Cross, S.J., Goldberg, J.M., 
Khatcheressian, J.L., Leighl, N.B., Perkins, C.L., Somlo, G., Wade, J.L., Wozniak, A.J., Armitage, J.O., Oncology, A.S. of C., 2015. J. Clin. Oncol. 33, 3199-3212.

Su, M., Ge, L., Kong, Q., Zheng, X., Ge, S., Li, N., Yu, J., Yan, M., 2015. Biosens. Bioelectron. 63, 232239.

Terstappen, L., DeGrooth, B.G., Visscher, K., VanKouterik, F.A., Greve, J., 1988. Cytometry 9, 39-43.

Varshney, M., Li, Y., 2009. Biosens. Bioelectron. 24, 2951-2960.

Virkki, R., Juven, T., Rikalainen, H., Svedstrom, E., Mertsola, J., Ruuskanen, O., 2002. Thorax 57, 438441.

Wang, X., Gartia, M.R., Jiang, J., Chang, T.-W., Qian, J., Liu, Y., Liu, X., Liu, G.L., 2015. Sensors Actuators B Chem. 209, 677-685.

Wei, F., Patel, P., Liao, W., Chaudhry, K., Zhang, L., Arellano-Garcia, M., Hu, S., Elashoff, D., Zhou, H., Shukla, S., Shah, F., Ho, C.M., Wong, D.T., 2009. Clin. Cancer Res. 15, 4446-4452.

Yang, L., Li, Y., Erf, G.F., 2004. Anal. Chem. 76, 1107-1113.

Yu, L.-Y., Xu, Z.-L., Shen, H.-M., Yang, H., 2009. J. Memb. Sci. 337, 257-265.

Zhang, D., Jiang, J., Chen, J., Zhang, Q., Lu, Y., Yao, Y., Li, S., Liu, G.L., Liu, Q., 2015. Biosens. Bioelectron. 70, 81-88.

Zhang, D., Liu, Q., 2016. Biosens. Bioelectron. 75, 273-284.

Zhang, Y., Bai, J., Wu, H., Ying, J.Y., 2015. Biosens. Bioelectron. 69, 121-127. 
(a)
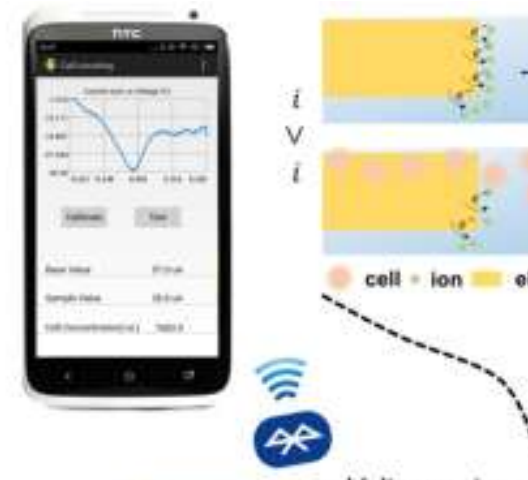

0

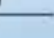

$i$
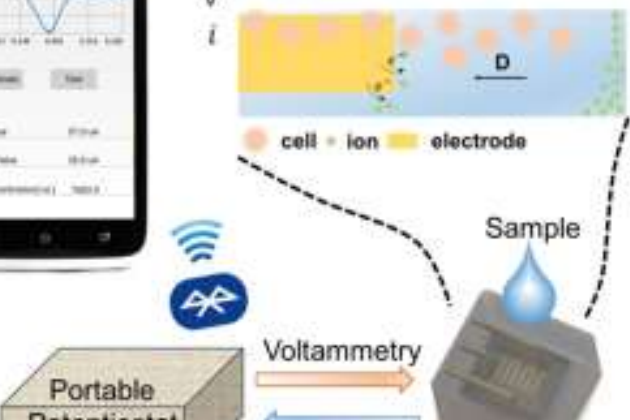

Voltammetry

Potentiostat

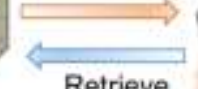

Retrieve

(b)

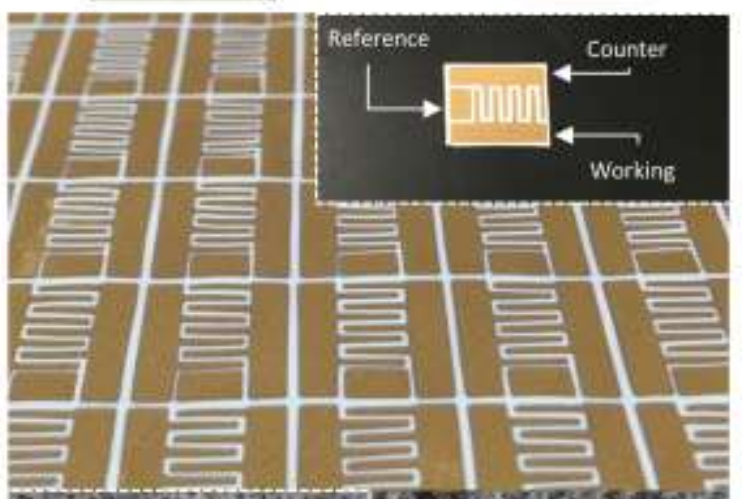

(c)

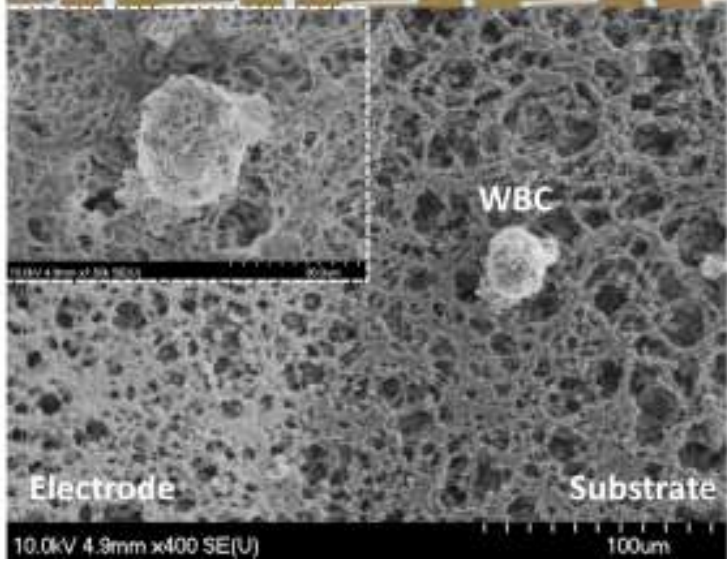

(d)

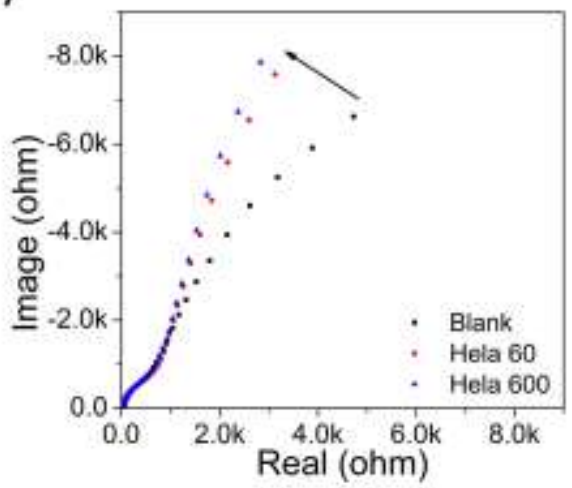

(e)

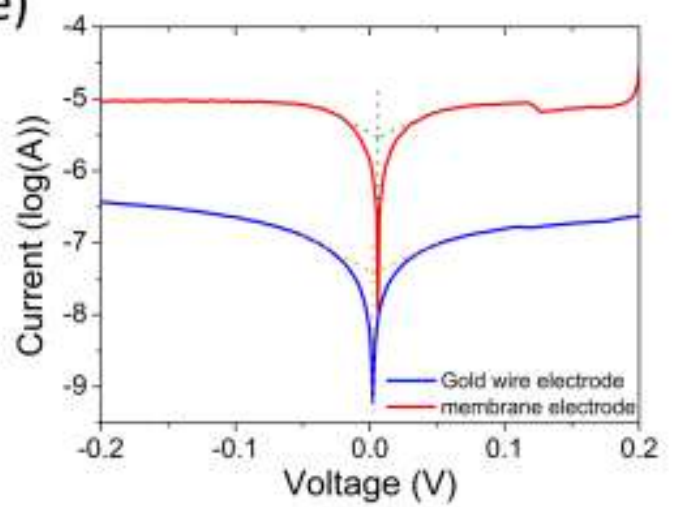

(f)

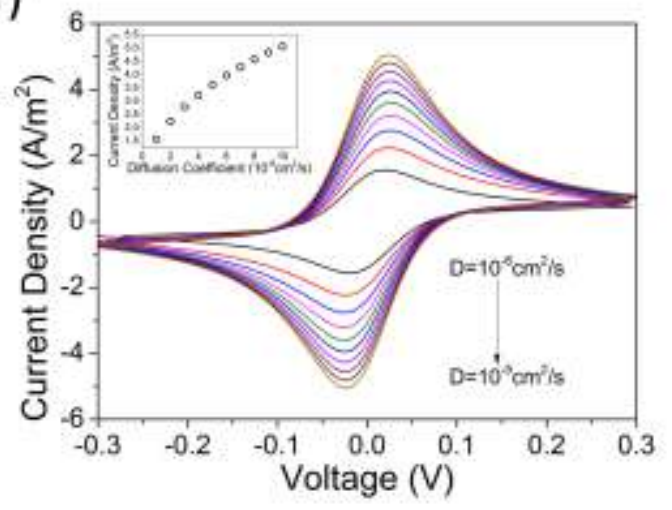

Fig. 1. Cell counting sensor design and principle. (a) System diagram and electrochemical principle for WBC count. D is diffusion coefficient. $i$ is the electrochemical current. (b) Gold three-electrodes on PVDF (polyvinylidene fluoride) membrane paper. (c) Scanning electron microscopy image of trapped WBC (white blood cell) on PVDF and microporous structure of PVDF membrane. (d) Nyquist plot of electrochemical impedance spectroscopy (EIS) showing the diffusion impedance upward bending by adding more Hela cells on the membrane electrode. The over-potential in EIS is $-0.5 \mathrm{~V}$ with frequency sweeping from $10 \mathrm{~Hz}$ to $100 \mathrm{kHz}$. (e) Tafel plot of the membrane electrode and wire electrode in $1 \mathrm{mM}$ $\left[\mathrm{Fe}(\mathrm{CN})_{6}\right]^{3-} /\left[\mathrm{Fe}(\mathrm{CN})_{6}\right]^{4-}$ and $10 \mathrm{mM}$ PBS at the scan rate of $0.1 \mathrm{mV} / \mathrm{s}$. (f) Simulation of cyclic voltammetry method at different diffusion coefficient in the environment of $1 \mathrm{mM}\left[\mathrm{Fe}(\mathrm{CN})_{6}\right]^{3-} /\left[\mathrm{Fe}(\mathrm{CN})_{6}\right]^{4-}$ and the inset is the peak current density change with diffusion coefficient. 
(a)

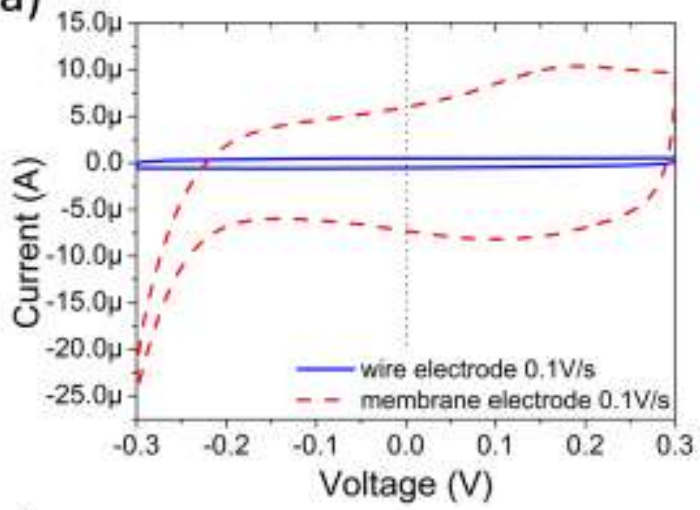

(c)

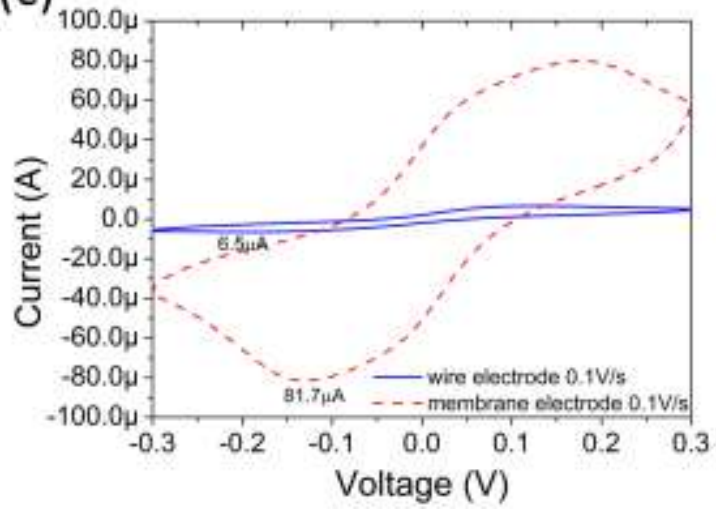

(b)
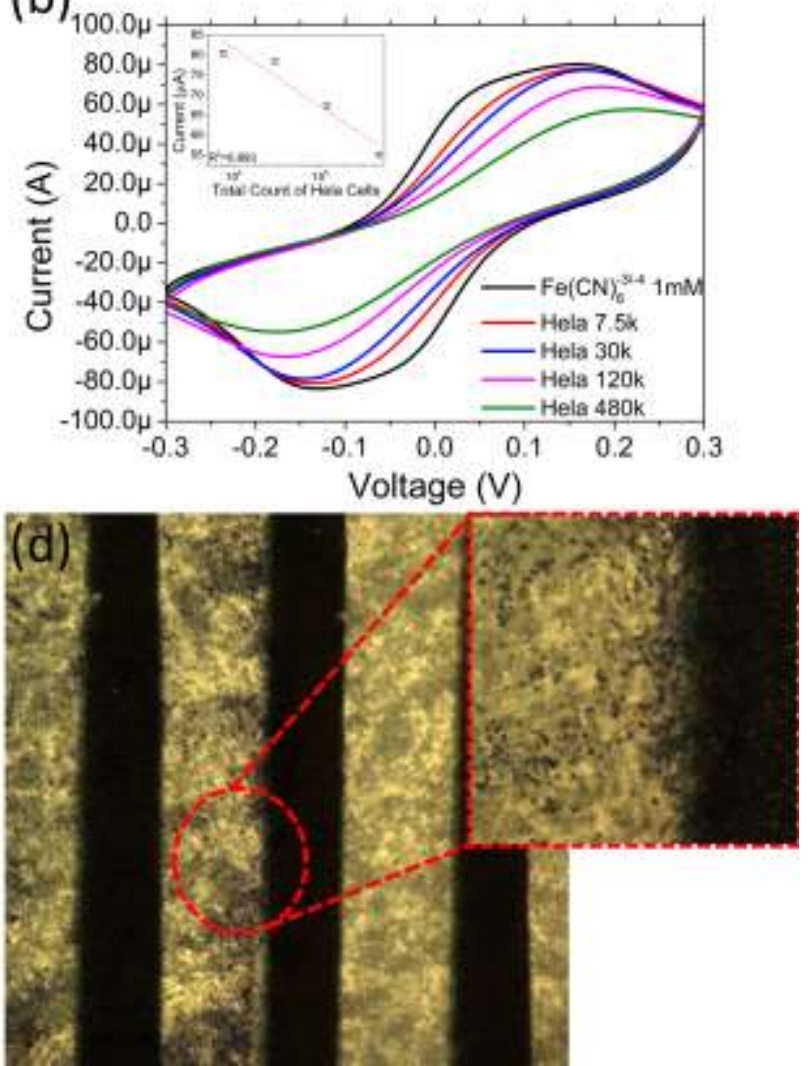

Fig. 2. Membrane Electrode Characterization. (a) Cyclic voltammetry test on wire and membrane electrode in electrolyte only $(10 \mathrm{mM}$ PBS) at the scan rate of $0.1 \mathrm{~V} / \mathrm{s}$. Double layer capacitance was estimated from current value at voltage 0. (b) Cyclic voltammetry for loading Hela cells at the amount of $7.5 \mathrm{k}, 30 \mathrm{k}, 120 \mathrm{k}$ and $480 \mathrm{k}$ with $1 \mathrm{mM}\left[\mathrm{Fe}(\mathrm{CN})_{6}\right]^{3-} /\left[\mathrm{Fe}(\mathrm{CN})_{6}\right]^{4-}$ and $10 \mathrm{mM}$ PBS in a volume of $10 \mathrm{uL}$. The inset shows the peak current calibration with total count of Hela cells. (c) Comparison of CV curves at the scan rate of $0.1 \mathrm{~V} / \mathrm{s}$ in $1 \mathrm{mM}\left[\mathrm{Fe}(\mathrm{CN})_{6}\right]^{3-} /\left[\mathrm{Fe}(\mathrm{CN})_{6}\right]^{4-}$ and $10 \mathrm{mM}$ PBS. (d) Microscopy image of trapped dyed Hela cells on membrane electrode. 
(a)

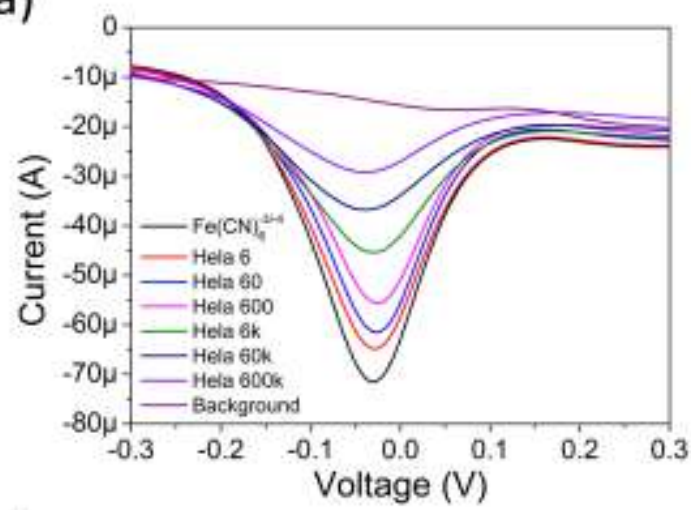

(c)

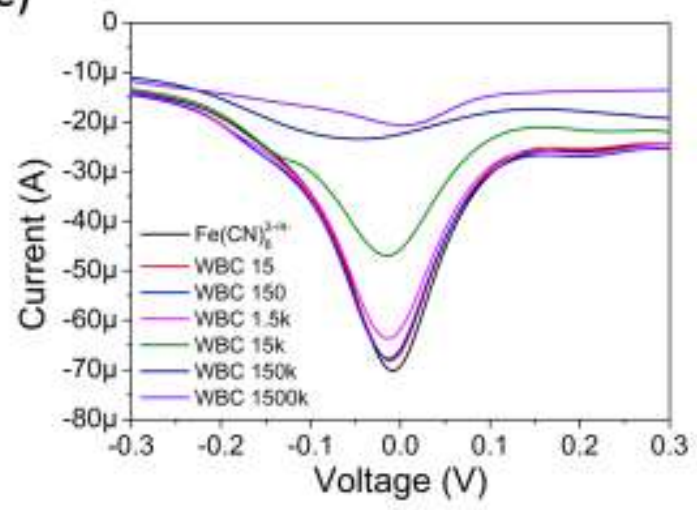

(b)

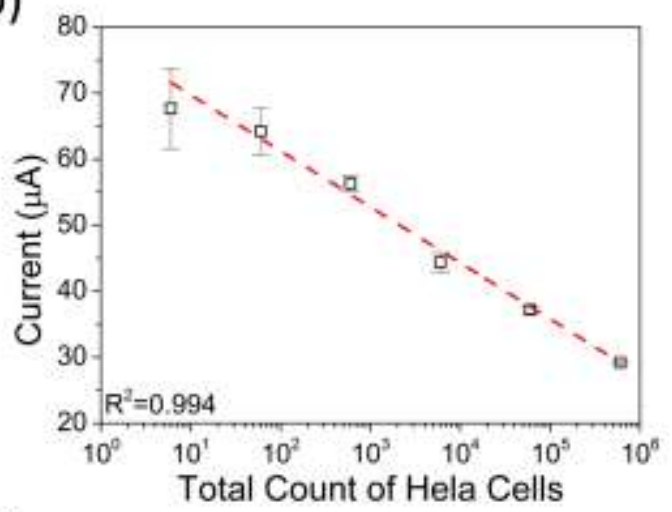

(d)

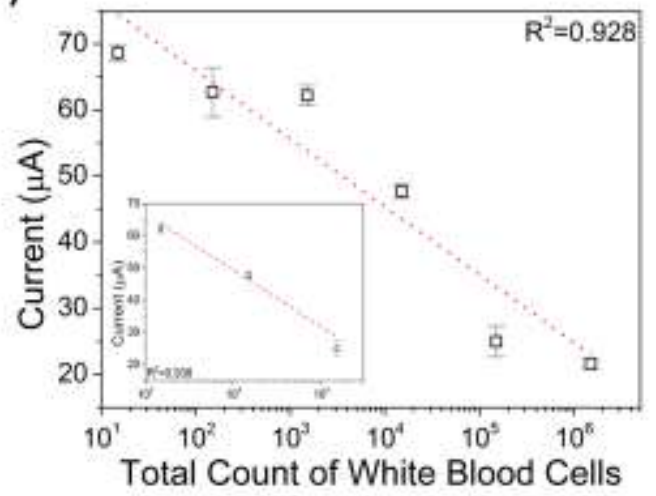

Fig. 3. Cell counting and calibration on membrane electrode using commercial potentiostat. (a) DPV reduction peak as the function of Hela cell numbers at the amount of 6 to 600k on membrane electrode. Background is just 10mM PBS. (b) Calibration curve of peak current as the function of Hela cell number based on 3 replicates. (c) DPV reduction peak after capturing white blood cells (WBC) at the amount of 15 to $1500 \mathrm{k}$ on membrane electrode. (d) DPV reduction peak as the function of WBC numbers at the amount of 6 to $600 \mathrm{k}$ on membrane electrode ( 3 replicates). The inset shows the peak current as the function of the WBC number in the range of $10^{3}$ to $10^{5}$. The DPV experiment was performed with incremental amplitude at $4 \mathrm{mV}$ and a period of $0.2 \mathrm{~s}$, as well as the pulse height at $50 \mathrm{mV}$ with $50 \mathrm{~ms}$ pulse width. All the calibration was made with the buffer of $1 \mathrm{mM}\left[\mathrm{Fe}(\mathrm{CN})_{6}\right]^{3-} /\left[\mathrm{Fe}(\mathrm{CN})_{6}\right]^{4-}$ in $10 \mathrm{mM} \mathrm{PBS}$. 

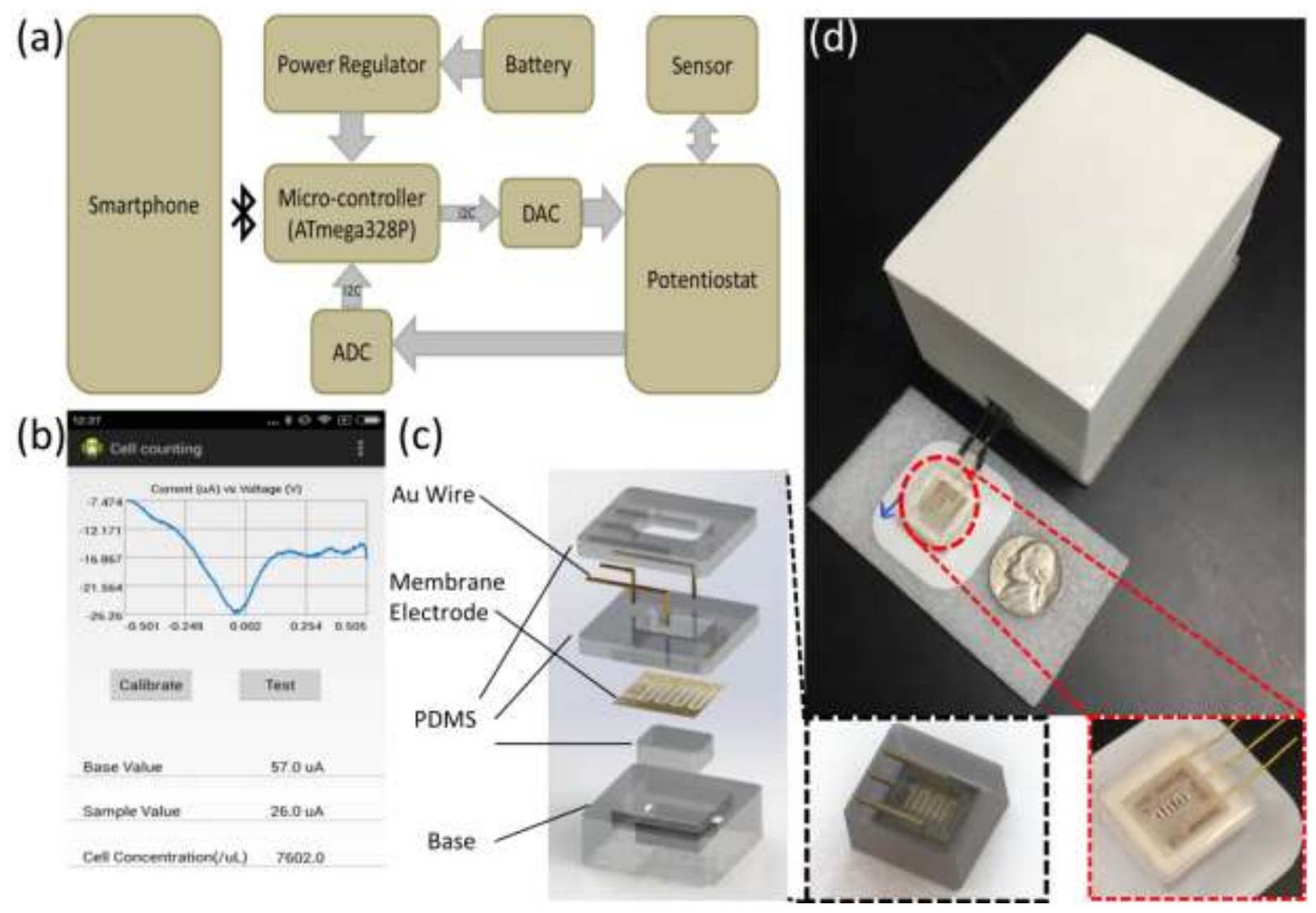

Fig. 4. Smartphone based electrochemical cell counting platform design. (a) Block diagram of the sensor electronics system. (b) Android App interface with received electrochemical sensing data through Bluetooth protocol plotted on smartphone screen. (c) Assembly and explosive view of the sensor package. (d) Whole package view with sensor assembly and electronic system. 
(a)

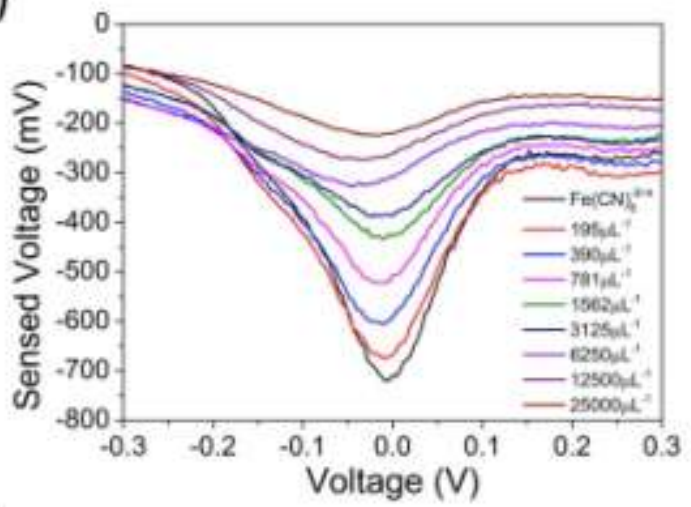

(c)

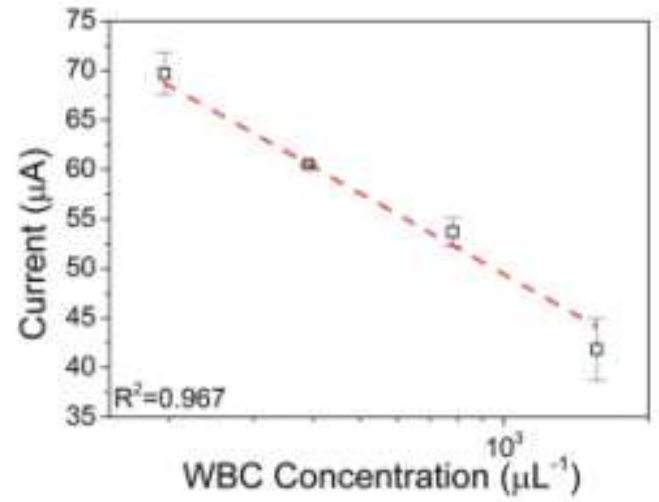

(b)

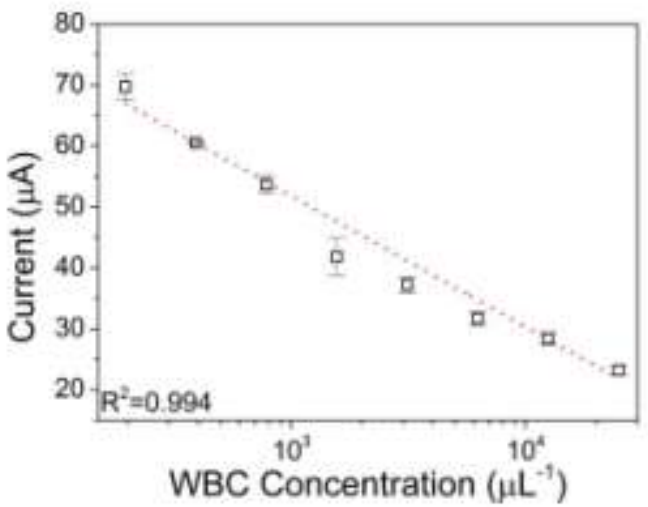

(d)

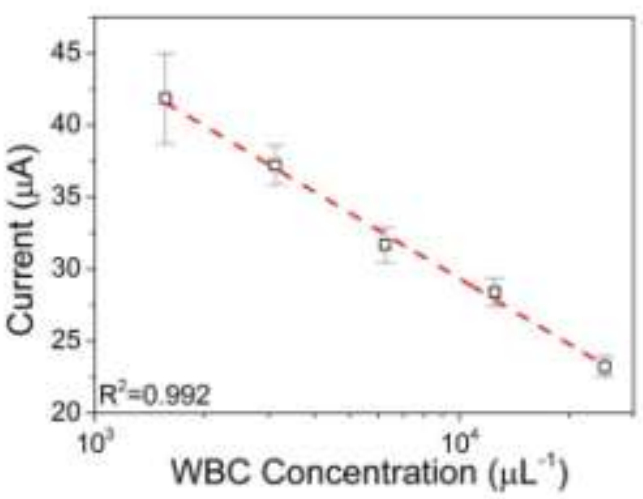

Fig. 5. WBC counting and calibration using smartphone electrochemical platform with PVDF membrane electrodes. (a) DPV reduction current peak corresponding to white blood cells at different concentrations in the volume of $10 \mu \mathrm{L}$. (b) Converted reduction peak current as the function of WBC concentration calibration with $\mathrm{R}^{2}=0.994$ (3 replicates). (c) Calibration curve for WBC concentration at low concentration range. (d) Calibration curve for WBC concentration at high concentration range. 
(a)

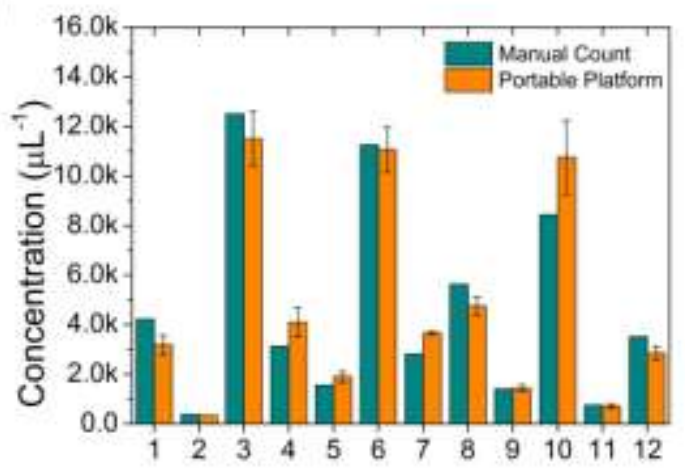

(b)

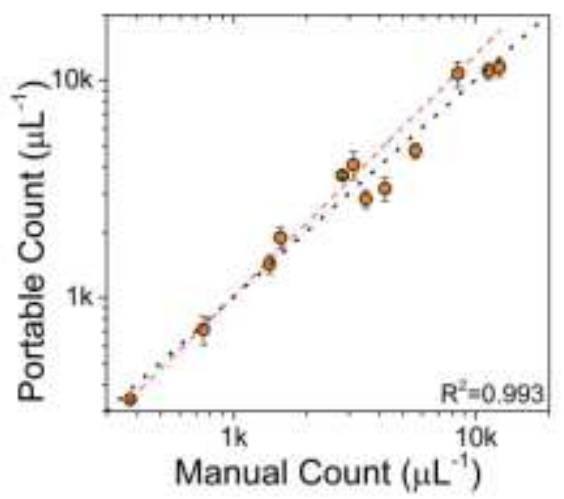

Fig. 6. Correlation study between the portable electrochemical cell counting method and manual counting. (a) Measurement of WBC with different concentration by manual count and portable electrochemical method respectively. (b) Comparison between portable electrochemical method and manual count method. The red dashed line is the linear regression fitting curve with $\mathrm{R}^{2}=0.993$. The black dot line is the $\mathrm{y}=\mathrm{x}$ line representing the perfect matching line. 